\title{
Determinants of Water Consumption Growth and Green Environment Trade-offs in Kombolecha Industrial Zone, Ethiopia
}

\author{
Tefera Eshete Kebede ${ }^{1}$, Chipo Mukonza ${ }^{2}$, Munyaradzi Chitakira ${ }^{2}$ \\ ${ }^{1}$ Department of Economics, Business and Economics College, Wollo University, Dessie, Ethiopia \\ ${ }^{2}$ School of Ecology and Human Sustainability, Department of Environmental Science, University of South Africa, Florida Campus, Calabash \\ Building, South Africa
}

Email address:

tefera_eshete@yahoo.com (T. E. Kebede), 50876791@mylife.unisa.ac.za (T. E. Kebede), chiponyam2@yahoo.com (C. Mukonza), chitam1@yahoo.com (M. Chitakira), mchitakira@gmail.com (M. Chitakira)

\section{To cite this article:}

Tefera Eshete Kebede, Chipo Mukonza, Munyaradzi Chitakira. Determinants of Water Consumption Growth and Green Environment Tradeoffs in Kombolecha Industrial Zone, Ethiopia. International Journal of Environmental Chemistry. Vol. 3, No. 2, 2019 , pp. 65-71. doi: $10.11648 /$ j.ijec.20190302.13

Received: July 20, 2019; Accepted: September 17, 2019; Published: December 26, 2019

\begin{abstract}
Water is life blood for people and firms to ensure their optimal benefits. This study identified the determinants of the water consumption growth and green environment tradeoffs at Kombolecha industrial zone, Ethiopia. This was due to Ethiopia is amongst drought affected and variable rainfall dependent country in eastern Africa. Above 80 percent of the populations are still engaged in agriculture sectors, which contributed 46 percent of the total GDP. However, factories and households are consumed groundwater resources without payment in Birr and quantity limits. As a result, the green environment lost its nature due to overconsumption of groundwater sources. This study employed binary logistic regression so as to identify the significant factors that determine the water consumption growth and green environment tradeoffs. In pursuit of this, study collected crossectional surveyed data from 338 households and 14 factories. Triangulated methodology was used to determine statistically significant factors. Accordingly, this study found that household's awareness about green mind adoption, technology use, marketing, and exchange and environment restoration was statistically significant and altered the water consumption growth and green environment tradeoffs by 0.000 values at the 5 confidence level. Moreover, household's poverty, consumption culture and behaviours, sensitivity and emotionality, ability and willingness to pay were also differently associated and statistically significant and affected the water consumption growth and green environment tradeoffs at the 5 percent significance level. This study, therefore, recommended that concerned institutions would be altered water consumption and recycling behaviours and consumption culture through charging groundwater payments and delivering trainings and capacityservices to recover the green environment in Kombolecha and at large in Ethiopia.
\end{abstract}

Keywords: Water Consumption, Green Environment, Tradeoffs, Kombolecha Industrial Zone

\section{Introduction}

Water resource is life blood and an economic good for household and firms to optimise their utility and profit respectively. However, the world faces twin challenges: expanding economic opportunities for a growing global population, and addressing environmental pressures that, if left unaddressed, could undermine our ability to seize these opportunities [1]. The industrialization in many countries in the past 100 years and the resource-based industrial activities have used up resources, mostly produced by developing countries. The tremendous industrial growth in the world economy and the current strong economic growth in some regions of the world, for example in Asia, some Latin American countries, Africa have generated a high demand for specific inputs [2]. Renewable as well as nonrenewable resources have been in high demand, and they are threatened with being depleted. In particular, natural resources, which are often extracted from developing countries, have significantly reduced the years to exhaustion for those 
resources [3]. The Intergovernmental Panel on Climate Change's (IPCC) predictions state that over the next decades, between 75 and 250 million people will be exposed to increased water stress due to climate change [6].

Ethiopia is among drought affected and variable rainfall dependent economy at eastern African countries. In the country, above 85 percent of the population are depending on agriculture sector, which contributed 46 percent of the national GDP [5]. The FDRE government has been envisaged national growth and transformation plan (GTP-1\&GTP- 2, 2015-2020) to transform the agriculture to manufacturing sectors. Industrial zone establishment and industry clustering strategies are major activities envisaged in major cities in Ethiopia [6]. Kombolecha city is among major industrial city in Ethiopia that government has been given due attention for industrial growth since 1996 e. c. Currently, the FDRE government clustred a new industrial zone on $1000 \mathrm{~m}^{2}$ in Kombolecha to enhance industrial growth in Amhara regional state. Kombolecah has $400 \mathrm{~km}$ distance to Djibouti border and comfortable for export and import of goods. Due to this, there are railway stations, dry port, universities and other transport and financial institutions. As a result, population density and factory growth are speeding up parallel to water resource consumption rise in Kombolecha city.

Water consumption demand also increasing due to rising population and factory' $\mathrm{s}$ consumption and production demand. Groundwater resource in Ethiopia is not still charged and hence consumers used freely without payments. Tap water consumption also increasing despite its payments per cubic metre payment is low compare to other Eastern African countries. Consequently, consumer's such, as households and factories are consumed groundwater resources without payment and discharged excess wastes to Borekna River without treatment. As a result the green environment is questionably addressed and in turn affected the household's living and working condition. This study, therefore, intended to determine the water consumption growth and green environment tradeoffs in Kombolecah industrial Zone, Ethiopia.

\section{Problem Statement}

Ethiopia is a highly rainfall dependent country and the economy relies on the agriculture sector. Exploitation of water resources might be generated a large economic benefit in the short term. However, in the long run, over-use of the groundwater is not only increased environmental degradation but also make economic growth and livelihood opportunities questionable. Ethiopia's flourishing economy is both a key driver to environmental degradation and at the same time, the economy is negatively affected by environmental problems that the country is phasing [7]. Particularly, water consumption put an immense pressure on resource degradation and environmental depletion. Consumers such as households and factories were key participants to erode the green environment in the growing industrial cities like

\section{Kombolecha.}

Balancing resource consumption and environmental tradeoffs become a burning agenda to rehabilitate eroded environment and mitigate climate changes [4]. On the other side, industrial and manufacturing growth, however, become a key pillar to transform the agriculture to industrial sectors in GTP-1 (2010 \& 2015) and GTP-2 (2016-2020). As a result, the new emerged firms were alarmingly increasing in various sectors in Kombolecha. The green environment was depleted by rising factories and household's water resource consumption without balancing the green environment problems, which resulted from inefficient consumption and waste recycling processes. As a result, the population growth and resource consumption have been shown tradeoff that treats the nature of green environment, which shared the notion green environment challenges today [5]. The groundwater, in fact, was seriously depleted by factories and households over consumption without payments per water quantity use, stakeholders have done nothing to protect the environment. This study, thus, identify the significant factors that determine the water resource consumption growth and green environment tradeoffs using a binary logistic regression model.

\section{Objectives}

This study general objective was determined factors that affect the water consumption growth and the green environment trade-offs in Kombolecha industrial zone.

\subsection{Specific Objectives}

This study specific objective includes:

1. Assessed household's perception and behaviours effect on water consumption growth and green environmenttrade-offs.

2. Identified significant factors affecting household's water consumption growth and green environment tradeoffs.

3. Evaluate household's awareness and willingness to pay the money for green mind, technology and consumption practices in meetingwater consumption and recycling efficiency.

\subsection{Research Questions}

This study research questions consisted:

What would be the effect of household's consumption perception and behaviours effect on water consumption growth and green environment Trade-offs?

What are the major statistically significant factors that affect the household's water consumption growth and the green environment tradeoffs?

\section{Significance}

Water is life blood to exist on earth. Ethiopia particularly Kombolecha is drought affect city for which consumerswere 
always faced water shortage. Since FDRE government envisaged agriculture industry transition national economic reform, water consumption demand increased parallel to factory and population density in cities like Kombolecha. Consumer's diverse and heterogeneous consumption behaviours and perceived water consumption activities triggered the green environment status. This study, therefore, would be contributed knowledge in the body of further research requirements that balance the water consumption and green environment problems. In addition to this, this study will be used for policy input for environment and water policy makers and practitioners in Ethiopia, researchers, student, who will be intended research and required further information about water consumption and green environment tradeoffs in Kombolecha.

\section{Methodology}

This study employed a descriptive research design to analyse the cross- sectional surveyed data during 2018. Households and factories were key participants of the study and provided data in Kombolecha. Different methods may be used for different purposes. Second, different data collection methods may be used to provide convergent evidence (a process referred to as triangulation). Based on this, this study, therefore, was used a triangulation methodology, which comprised of both qualitative and quantitative methods. Quantitative methods markedly used descriptive statistics and binary logistic regression model. Latest version of SPSS20 and stata14 were used to identify significant factors during data analysis.

This study mainly targeted 'Kebele' four administration ('Kebele' here refers the lowest administration unit in Ethiopia) and the industrial Zone where people and factories are densely populated. The population frame was 3252 households, who composed of households that consumed water resources and recycled wastes differently. Nonetheless, the target population was sample households, who provided information in the period of primary data collection in the study area.

Based on household's complex socio-demographic characters and consumption patterns, the target population was divided into mutually exclusive groups and classified into four major categories: namely, factory employees (1537), consumers excluding factory employee (1265), suppliers (450) and service providers (125) such as hotel, garage, café services etc. To get accurate information from each category, stratified random sampling techniques were applied to sample households. Household's category served as a stratum. Out of each stratum, individual households were selected randomly to give each household an equal chance of being selected.

Out of four categories of households, the total sample households from all stratawasn $n_{i}=n_{1}+n_{2}+n_{3}+n_{4}$. Accordingly, sample households were factory employee $\left(n_{1}=154\right)$, consumers $\left(\mathrm{n}_{2}=126\right)$, suppliers $\left(\mathrm{n}_{3}=55\right)$, and service provider $\left(n_{4}=4\right)$ from each stratum. Hence, 338 sample households from Kombolcha industrial zone were selected to gather data using semi structured questionnaires and interview.

The questionnaire and interview schedules consist of both open and close ended questions. The collected data was analysed and regressed using a binary logistic regression model and assumption, which proposed that the dependent variable would have binomial responses [8].

Along with this, variables namely, household's income, employment status, education level, perception, attitudes, behaviour, ability and willingness to pay, culture, awareness, sensitive and emotionality were major explanatory variables included in the working hypothesis. The dependent variable was respondent's water consumption growth and green environment tradeoffs (CONENVTRD). This tradeoff would be affected by household's employment status (HHEMP), perception (HHPRC), behaviour (HHBEH), Attitudes (HHATT), Awareness (HHAWR), Income (HHINC), Education level (HHEDU), ability to pay (HHABI), willingness to pay (HHWPA), and etc. Meanwhile, the study formulated a relationship between the explained and explanatory factors.

In other words, resource consumption growth and green environment tradeoff (CONEVTRD) is a function of independent variables in the following ways:

CONENVTRD $=\mathrm{f}($ HHEMP, HHPRC, HHBEH, HHATT, HHAWR, HHINCOM, HHEDU, QWA, HHSEMO, HHABI, HHWPA, and etc)

Where;

1. CONEVTRD=Water Resource Consumption growth and green Environment Tradeoff.

2. EMP, PRC, BEH, ATT, AWR, INCOME, EDU, QWA, SEMOE, ABP, WPA, SOW respectively presents household's employment, perception, behavior, attitude, awareness, income, education level, quantity of water consumed and recycled, sensitivity and emotionality, ability and willingness to pay.

After specifying this tradeoff function in linear form including error term $\left(\mathrm{e}_{\mathrm{i}}\right)$, it was formulated a multiple linear regression model as follow:

CONEVTRD $=\beta_{0}+\beta_{1}$ HHEMP $+\beta_{2}$ HHPRC $+\beta_{3}$ HHBEH + $\beta_{4}$ HHATT $+\beta_{5}$ HHAWR $+\beta_{6}$ HHINC $+\beta_{7}$ HHEDU $+\beta_{8}$ QWA $+\beta_{9}$ QWAS $+\beta_{10}$ HHSEMOE $+\beta_{11}$ HHABP $+\beta_{12}$ HHWPA

$$
+\ldots+\text { and etc }+\mathrm{e}_{\mathrm{i}}
$$

Where, it is possible to present $C O N V E T R D=Y_{i}$ and the explanatory factors $=\mathrm{X}_{\mathrm{i}}$. The model would be;

$$
\mathrm{Y}_{\mathrm{i}}=\beta_{0}+\beta_{1} \mathrm{X}_{1}+\beta_{2} \mathrm{X}_{2}+\beta_{3} \mathrm{X}_{3}+\beta_{4} \mathrm{X}_{\mathrm{i}}+\ldots+\mathrm{e}_{\mathrm{i}}
$$

The rationality of constructing binary logistic regressions was the fact that it helped to hold multiple factors and showed association between binary response factors and measurements. Based on the constructed model, which shows association between dependent and independent factors, hypothesis for each explanatory variable was proposed and represented by $\mathrm{H}_{\mathrm{i}}$. Where, $\mathrm{i}=1,2 \ldots, \mathrm{n}$.

Variable proposition and Hypothesis:

H1: Household's perception has no significant effect on water resource consumption to protect environment. 
H2: Household's consumption Behaviour has no significant effect on water consumption to protect environment.

H3: Household's attitude has no significant effect on water resource consumption to protect environment.

H4: Household's Awareness has no significant effect on water resources consumption to protect environment.

H6: Household's education level has no significant effect on water resource consumption to protect environment protect environment.

H7: Household ability and willingness to pay money has no significant effect on water resource consumption to protect environment.

\section{Results}

In this study context, green environment referred the concerns of environmental conservation and improved the health and quality of the environment by balancing the water resource consumption and recycling processes. Environment problems were severely affected people living condition along with Borkena river edges. In the rationality of this study, the green environment is paramount that entails a favourable condition for households to live and work healthily. It was investigated that factories and households were keenly disrupting the green environment status by inefficient water consumption and recycling processes. To elaborate on this issue, 338 households were participated and questioned whether the green environment problems were existed or not. Accordingly, out of the total households, 236 $(68.9 \%)$ respondents agreed about the presence of the green environment problems. However, the remain 106 (31.1\%) respondents were not agreed about the existence of the green environmental problems in Kombolecha.

Table 1. Green Environmental Problems in Kombolecha.

\begin{tabular}{lll}
\hline Environmental problems & Respondents & Percent \\
\hline Borkena river/water/pollution & 139 & 41.1 \\
air pollution & 118 & 34.9 \\
living environment pollution & 70 & 20.7 \\
working environment pollution & 11 & 3.3 \\
Total & 338 & 100.0 \\
\hline
\end{tabular}

Source: Survey Results, 2017.

Environmental problems were categorized into river, air, living and working environment pollution in Table 1. Among mentioned environmental problems, out of 338 sample populations, $139(41.1 \%)$ respondents agreed that the water resources, particularly, the Woreqa and Borkena rivers, which pass via the centre of a city, were polluted by factories and household's waste discharges. Despite waste quantities were varied across the consumer's emission, none of them was treated the liquid waste to protect the living and working environment. Out of the total population, nevertheless, 118 (34.9\%) and 70 $(20 \%)$ respondents agreed that the living and air pollution were major environmental problems respectively. The federal government of Ethiopia clustered 75 hectares of land and launched a new industrial zone in Kombolecha (Kombolecha communication office, 2017). However, there was no, yet, projected groundwater consumption and waste recycling regulatory procedures that reduce the river's pollution and make the living and working condition healthy.

This study binary logistic regression showed that the household's awareness to practice the green mind (Awgrnmin) was positively improving the trade-offs between consumption growth and green environmental problems. In other words, the household's awareness to adopt the green mind was among the key factor that predisposed the tradeoffs between consumption growth and green environmental problems. In addition to this, the household's awareness headed of green buying (AWgrnbuy) and technology use (AWgrntech) were positively affected the consumption growth and green environment trade-offs. For the most part, consumer's awareness to use a green technology (AWgrntech) was drastically altered the consumption growth and green environment trade-offs by 0.004 values at the 5 percent significance level. However, the respondent's awareness about the green product consumption (AWgrnpco) and jobs look for (Awgrnjob) were not principally affected the consumption growth and green environmental trade-offs at the same level of significance.

Table 2. Green Awareness effect on COENVTRD.

\begin{tabular}{|c|c|c|c|c|c|c|}
\hline \multicolumn{7}{|l|}{ Robust } \\
\hline COENVTRD & Coef. & Std. Err. & $\mathbf{Z}$ & $\mathbf{P}>|\mathbf{z}|$ & \multicolumn{2}{|c|}{ [95\% Conf. Interval] } \\
\hline Awgrnmin & 3.41829 & .5883349 & 5.81 & $0.000 *$ & 2.265174 & 4.571405 \\
\hline Awgrnpco & -1.903145 & .6648474 & -2.86 & $0.004 *$ & -3.206222 & -.600068 \\
\hline Awgrnbuy & -.7616682 & .4676024 & -1.63 & 0.103 & -1.678152 & .1548157 \\
\hline Awgrntec & -1.862999 & .752474 & -2.48 & $0.013^{*}$ & -3.33782 & -.3881765 \\
\hline Awgrnjob & .7726067 & .4415615 & 1.75 & 0.080 & -.092838 & 1.638051 \\
\hline Awgrnenv & 1.424179 & .300324 & 4.74 & $0.000^{*}$ & .8355544 & 2.012803 \\
\hline
\end{tabular}

*indicates significant factors at the 95 confidence level.

Source: Survey Results, 2017. 
Table 2 exemplifies the household's green awareness and its effect on water consumption growth and green environment trade-offs. Successively, the household's awareness to adopt a green mind (Awgrnmin) by 0.000 ; consumption (Awgrnprco) with 0.004; technology (Awgrntech) with 0.013 and environment (Awgrnenv) with 0.000 values were found statistically significant and influenced the consumption growth and green environment trade-offs at the 5 percent significance level. However, this study found that the household's awareness about adopting the green mind were strongly affecting the trade-offs between consumption growth and green environment trade-offs (COENVTRD) with 0.000 values at the 5 percent significance level.

In addition to this, the household's socio-demographic characters were associated and coupled with the trade-offs between resource consumption growth and the green environmental problems. For example, this study binary logistic regression depicted that the household's birthplace by 0.003 values; housing ownership with 0.045 and health status with 0.000 values werestatistically significant and influenced their awareness to practice the green mind at the 5 percent significance level. However, the household's age by 0.045 , education level with 0.046 and housing ownership with 0.038 values were statistically significant and importantly created a difference to practise the green consumption and recycling processes. Moreover, the household's employment status by 0.000 ; types of working sectors with 0.011 and health status with 0.000 values were calculated statistically significant and strongly shaped their awareness and experience to the green consumption.

Table 3. Household's Perception Effect on COENVTRD.

\begin{tabular}{|c|c|c|c|c|c|c|}
\hline COENVTRD & Coef. & Std. Err & $\mathbf{Z}$ & $\mathbf{P}>|\mathbf{z}|$ & \multicolumn{2}{|c|}{ [95\% Conf. Interval] } \\
\hline HHPLWENI & -.6335702 & .2717764 & -2.33 & $0.020 *$ & -1.166242 & -.1008982 \\
\hline HHPGrnco & .745936 & .2937399 & 2.54 & $0.011 *$ & .1702164 & 1.321656 \\
\hline HHPgrnpr & -.8574688 & .3074394 & -2.79 & $0.005^{*}$ & -1.460039 & -.2548986 \\
\hline HHPgrnMk & .0615853 & .2636859 & 0.23 & 0.815 & -.4552295 & .5784001 \\
\hline HHPGTECH & -.4822763 & .2521824 & -1.91 & $0.056^{*}$ & -.9765448 & .0119922 \\
\hline HHPGindu & -.469131 & .2546109 & -1.84 & 0.065 & -.9681591 & .0298972 \\
\hline
\end{tabular}

NB: * indicates significant factors at 95 confidence level.

Source: Survey Results, 2017.

Table 3 illustrates the household's perception effect on resource consumption growth and the green environment trade-offs (COENVTRD). Based on this, the respondent's perception to protect the green living and working environment (HHPLWENI) by 0.02 , production (HHPgrnpr) with 0.005, consumption (HHPGrnco) with 0.011, and technology (HHPgrnMk) use by 0.05 values were statistically significant and affected the resource consumption growth and green environment trade-offs at the 5 percent significance level. However, the household's perception to exchange at the green market was not found statistically significant and affected the consumption growth and green environment trade-offs. Nonetheless, the household's green perception to resilient the green industrial zone (HHPGindu) was negatively allied with the water consumption growth and green environmental trade-offs. For example, when the household' perception to green an industrial zone (HHPGindu) was increased by a unit, the resource consumption growth and green environment trade-offs was reduced by 46.9 percent at the 5 percent significance level.

In addition, the household's perceptions were measured by good, bad, fair, not good and unfair response. The household's, who have a bad perception about the green environment, was significantly interconnected to their poverty status by 0.043 , working sector types with 0.000 and health problems with 0.008 values at the 5 percent significance level. In other words, the respondent's poverty; working types and health status were determinant factors that lead them to have a bad perception about the green environment. The respondents, who have health problems, worked in the factory, and became poor, have a bad perception to resilient the green environment. However, nonpoor respondents have a good perception to resilient the green environment. Poor respondents were found psychologically deprived; lacked confident and became voiceless to protect groundwater degradation and resilient the depleted environment in Kombolecha.

Table 4. Household's Green Behaviours Effect on CONVETRD.

\begin{tabular}{|c|c|c|c|c|c|c|}
\hline \multirow{2}{*}{$\begin{array}{l}\text { COENVTRD } \\
\text { HHBWgrnc }\end{array}$} & \multirow{2}{*}{$\begin{array}{l}\text { Coef. } \\
1.391093\end{array}$} & \multirow{2}{*}{$\begin{array}{c}\text { Std. Err. } \\
.4142593\end{array}$} & \multirow{2}{*}{$\frac{\mathbf{Z}}{3.36}$} & \multirow{2}{*}{$\frac{\mathbf{P}>|\mathbf{z}|}{0.001^{*}}$} & \multicolumn{2}{|c|}{ [95\% Conf. Interval] } \\
\hline & & & & & .5791599 & 2.203026 \\
\hline HHBWeco & -.4847289 & .3498434 & -1.39 & 0.166 & -1.170409 & .2009516 \\
\hline HHBwNip & 1.384769 & .375413 & 3.69 & $0.000 *$ & .6489733 & 2.120565 \\
\hline HHBwrkig & -.9832376 & .3191808 & -3.08 & $0.002 *$ & -1.608821 & -.3576547 \\
\hline HHBwfutg & -.5949729 & .299767 & -1.98 & $0.047^{*}$ & -1.182505 & -.0074403 \\
\hline
\end{tabular}




\begin{tabular}{llllll}
\hline COENVTRD & Coef. & Std. Err. & Z & P>|z| & [95\% Conf. Interval] \\
\hline HHBwenvp & -.0590846 & .2898647 & -0.20 & 0.838 & -.627209 \\
cons & 1.274535 & & & & \\
\hline
\end{tabular}

NB: * indicates significant factors at the 95 percent confidence level.

Source: Survey Results, 2017.

Table 4 illustrates the household's consumption behaviours that attempted to reduce an economic aspect (HHBWgrnc) but increase the living environment protection (HHBWlivp) were negative and notably coupled with the trade-offs between water consumption growth and the green environment problems. Accordingly, the binary logistic regression revealed that the household's consumption behaviours aimed to reduce economic costs (HHBgrnco) with 0.001 ; neighbour's environment (HHBwNip) with 0.000 ; living environment (HHBlivp) with 0.048, and the working environment protection (HHBwrking) with 0.002 values were statistically significant and importantly influenced the consumption growth and green environment trade-offs at the 5 percent significance level. However, the household's consumption behaviours, which concerned the neighbours environment protection, were strongly affected the water consumption and recycling efficiency. The finding of the study was not similar to Makower (2009), who argued that consumers may be interested in greening, but cannot identify it [9). This study found that consumer's green resource consumption behaviour was different to protect their living, working and the surrounding environment at the 95 percent confidence level.

Table 5. Household's Ability and Willingness Effect on COENVTRD.

\begin{tabular}{|c|c|c|c|c|c|c|}
\hline COENVTRD & Coef. & Std. Err. & $\mathbf{Z}$ & $\mathbf{P}>|\mathbf{z}|$ & \multicolumn{2}{|c|}{ [95\% Conf. Interval] } \\
\hline HHablity & .4656368 & .2169348 & 2.15 & $0.032 *$ & .0404523 & .8908213 \\
\hline HHwiling & -.3139389 & .271906 & -1.15 & 0.248 & -.8468648 & .218987 \\
\hline watrconl & .6537209 & .1753818 & 3.73 & $0.000 *$ & .3099789 & .9974628 \\
\hline $\begin{array}{l}\text { HHFRcult } \\
\text { cons }\end{array}$ & $\begin{array}{l}1.302826 \\
-3.042286\end{array}$ & .3207633 & 4.06 & $0.000^{*}$ & .674142 & 1.931511 \\
\hline
\end{tabular}

NB: * indicates significant indicators at the $95 \%$ confidence level.

Source: Survey Results, 2017.

Table 5 computes the household's ability and willingness effect on the resource consumption growth and green environment trade-offs (COENVTRD). Accordingly, household's ability to pay money (HHability) by 0.32 values, water consumption limit (HHWatrconl) by 0.000 values and consumption culture (HHFRRcult) by 0.000 values were statistically significant and determined the trade-offs between water consumption growth and green environment trade-offs. This result is consistent with findings in [13]. Above all, this study identified that respondent's ability to pay the money was altered by their green consumption with 0.012 values and production with 0.021 values at the 5 percent significance level. This study, nonetheless, identified that the respondent's consumption culture (HHFCULT) and water quantity limit (Watrconl) by 0.000 values were strongly affected their willingness to pay the money and in turn, influenced the water resource consumption growth and green environment trade-offs. This study result was supported by findings done so far $[14,15]$. The household's sensitivity and emotionality for economic costs (HHSSEco), living environment (HHSSLIV), health (HHHLTH) and neighbour's environment protection (HHSSNIB) were influenced their willingness to pay the money.

\section{Conclusion and Recommendation}

This study identifieddeterminant factors, which significantly affected the water resource consumption growth and green environmental tradeoffs in Kombolecha city,
Ethiopia. In order to do so, a triangulated research method, which consisted both quantitative and quantitative methods, used to identify factors, for instance, the household's perception and behaviours headed for adopting a green mind, technology and water use that cleared the water consumption growth and green environment tradeoffs. The household's perception, consumption behavior, awareness, attitude, sensitive and emotionality, willingness and ability to pay the money were measured with regards to their experience to adopt a green mind, technology and job use during the water consumption and recycling processes. This study shared the notion of the green nudge, motivation and consumer behaviour of people $[10,11]$. Meanwhile, this study devised a binary logistic regression to measure the effects of each factor on the water consumption growth and green environment tradeoffs.

The binary logistic regression and Environmental Kuznet Curve Model computed that there was a wide green water consumption behavioural inequality between households so as to adopt the green mind, technology use, market, and jobs that balanced the consumption growth and green environmental tradeoffs. Moreover, respondent's perception towards green consumption was influenced by their family size and income level. For example, it was computed that 247 (73\%) households have little perception to practice green consumption but reduced the green environment tradeoffs. This finding was quite different from study results in [12]. On the other hand, this study found that 173 (51.2\%) male and $35(10.4 \%)$ male and female households were not 
behaved well about green technology use. However, 213 $(63 \%)$ of respondent's consumption behaviours were aimed at reducing the economic cost compared to the social and environment costs. Out of the total households, 268 (79.3\%) respondents were disagreed that their water consumption behaviours were not environmental friend. However, household's housing ownership significantly altered the water consumption growth and green environment tradeoffs by 0.013 values at the 5 percent significance level. This was due to the household's sensitivity and emotionality for their own housing and ownership was significantly shaped the water consumption behaviour by 0.027 values at the same level of significance.

The household's green awareness to adopt the green mind by 0.002 values; living environment with 0.000 values, and technology use with 0.004 values were also positively associated and statistically influenced the consumption growth and green environment tradeoffs at the 5 percent significance level. The household's awareness about the green production was not influenced the consumption growth and green environment tradeoffs in Kombolecha. However, the household's perception towards practicing the green living and working environment (HHPLWENI) by 0.02 values; production (HHPgrnpr) by 0.005 values; consumption (HHPGrnco) by 0.011 values; technology (HHPgrnMk) by 0.05 values were statistically significant and affected the consumption growth and green environment tradeoffs. However, the household's perception about the green market was not affecting the resource consumption growth and green environment tradeoffs.

In addition to this, the household's consumption behaviours, which considered the economic cost reduction by 0.000 value; working environment by 0.000 values; living environment with 0.007 values and neighbour's environment protection by 0.000 values were found statistically significant and determined the water consumption growth and green environment tradeoffs at the 5 percent significance level. The household's consumption behaviour concerning to keeping the future water demand by 0.047 values and health protection (HHSSLTH) by 0.033 values were found significantly determined the water consumption growth and green environmental tradeoffs (CONVETRD) at the same level of significance. However, the respondent's consumption culture was significantly and strongly influenced the sensitive and emotional to behave green consumption by 0.000 value at the 5 percent level of significance. This study, therefore, incorporated the consumer culture (social aspect) into an eco-efficiency and developed a socio-eco efficiency framework. However, the household's willingness to pay the money was statistically significant and determined their sensitive and emotionality to apply the socio-eco efficiency framework by 0.000 values. This, study, therefore, recommended that consumers should be paid for groundwater consumption to resilient the green environment through balancing consumption growth and green environment tradeoffs in Kombolecha and at large in Ethiopia.

\section{References}

[1] OECD 2011b: Towards Green Growth - A summary for policy makers. OECD, Paris.

[2] (UNIDO, 20011 \& Kombolecha municipal, 2014): Green Industry: Policies for Supporting Green Industry: Vienna. Accessed: www.unidio.org/green industry.

[3] UNEP, 2011: Towards a Green Economy: Pathways to Sustainable Development and Poverty Eradication: A Synthesis for Policy Makers. Accessed: www.unep.org/greeneconomy.

[4] OECD 2012b: Greening Development: Enhancing Capacity for Environmental Management and Governance. OECD, Paris.

[5] Uno S 2011: Urban Development and The Environmental Challenges "Green" Systems Considerations: Issue Paper Commissioned By The European Commission. The Stockholm Resilience Center: Stockholm University.

[6] Greiner A and Semmler W 2005: "Economic Growth and Global Warming: A Model of Multiple Equilibria and Thresholds." Journal of Economic Behaviour and Organization. 57: 430-447.

[7] Valerie N 2011: Water Security in Ethiopia: Risks and Vulnerabilities' Assessment. The Intergovernmental Panel on Climate Change's (IPCC) $4^{\text {th }}$.

[8] Mark et al., 2002: global water outlook to 2025: Averting an Impending Crisis. International Water Management Institute (Iwmi). International Food Policy Research Institute. ISBN 089629-646-6. Washington: USA.

[9] Gujarati D 2004: Basic Econometrics, Third Edition. New York: Mcgraw-Hill.

[10] Emelie C and Anders E 2013: Environmental and Climate Change Policy Brief, Ethiopia. Goterbrg University Swedish Embassy, Ethiopia.www.sidaenvironmenthelpdesk.se.

[11] Lorenzo (2013): Lorenzo C 2013: Finding a Green Nudge: Moral Motivation and Green Behaviour Working Paper Last Revision. Paris School Of Economics, Panthéon-Sorbonne: France.

[12] EEA 2013: Environmental Indicators Report, 2013: Natural Resources and Human Well-Being in A Green Economy. Copenhagen, Denmark.

[13] Smith, 2013: Assessing The Impact Of Greening Perceptions On Consumer Purchasing Behavior: International Journal Of Economics And Management Sciences Vol. 2: No. 11: Pp. 94107. Department Of Business Management: Nelson Mandela Metropolitan University: South Africa.

[14] Tefera E, 2018, development of resource model for greening environmental resilience: an application of socio-eco efficiency framework in Kombolecha industrial zone. $\mathrm{PhD}$ thesis, college of Agriculture and environmental science, University of South Africa. South Africa.

[15] Sailing, et al. (2013): How to measure social impacts? A socio-eco efficiency analysis by SEEBALANCE Method. Karlsruhe university, Jena university and Oko-institute. Germany. 